\title{
Symmetric research joint ventures: Cooperative substitutes and complements
}

\author{
Dermot Leahy $^{\mathrm{a}}$, J. Peter Neary ${ }^{\mathrm{a}, \mathrm{b}, *}$ \\ ${ }^{\mathrm{a} U n i v e r s i t y ~ C o l l e g e ~ D u b l i n, ~ B e l f i e l d, ~ D u b l i n ~ 4, ~ I r e l a n d ~}$ \\ ${ }^{\mathrm{b}} C E P R$, London, UK
}

Received 24 May 2004; received in revised form 23 December 2004; accepted 21 January 2005

Available online 24 May 2005

\begin{abstract}
We introduce the concepts of cooperative substitutes and complements, and use them to explain when all firms in a research joint venture will choose equal levels of R\&D. We show that the secondorder conditions for a symmetric optimum take a particularly simple form, ruling out both excessive cooperative substitutability and excessive cooperative complementarity, and nesting conditions already derived in the literature. Finally we apply our results to the comparison between cooperative and non-cooperative R\&D, and show in an important special case that asymmetric outcomes are only optimal for a very limited range of parameter values.
\end{abstract}

(C) 2005 Elsevier B.V. All rights reserved.

JEL classification: D43; L13; L40

Keywords: Bertrand and Cournot competition; Cooperative substitutes and complements; R\&D; Research joint ventures; Strategic trade and industrial policy

\section{Introduction}

In recent years an extensive theoretical literature has developed with important implications for several policy debates, including the desirability of research joint

* Corresponding author.

E-mail addresses:dermot.m.leahy@ucd.ie (D. Leahy), peter.neary@ucd.ie (J.P. Neary). 
ventures, R\&D subsidies, and strategic trade and environmental policy. This literature considers two-stage oligopoly games in which first-stage actions affect second-stage profits, and has frequently focused on symmetric equilibria where identical agents take identical actions. The case where firms are identical ex ante is a natural starting point for exploring these issues, since there is often no obvious way of justifying initial differences between firms. To many authors it has seemed equally natural to focus on symmetric outcomes, in which firms are identical ex post.

More recently, however, doubts have been expressed concerning the appropriateness of ex post symmetry in such models. Amir and Wooders (1998), following Henriques (1990), show in a non-cooperative game with R\&D followed by output competition that symmetric equilibria may be unstable, and they interpret such instability as implying that the outcome will be asymmetric. Van Long and Soubeyran (1999) explore the conditions under which symmetric firms which engage in a research joint venture will choose an asymmetric pattern of R\&D. Most notably, Salant and Shaffer (1999) draw on the insight of Bergstrom and Varian (1985) that a mean-preserving increase in the variance of marginal costs raises industry profits in Cournot competition. Building on this, they show that firms may have an incentive to deviate from a symmetric outcome. Referring in particular to the literature on $R \& D$ cooperation stemming from d'Aspremont and Jacquemin (1988), they assert that "this entire literature assumed (incorrectly) that it is joint-profit maximizing for the cartel to treat identical members equally." (Salant and Shaffer (1999, p. 586).) They also claim that there is a presumption that equilibria in such models will be

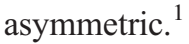

Notwithstanding these contributions, there does not appear to be a precise statement in the literature of the conditions under which cooperative equilibria will be ex post symmetric. The principal objective of this paper is to derive the exact restrictions which imply that symmetry is optimal, and to compare them with conditions previously derived by d'Aspremont and Jacquemin and Salant and Shaffer. In order to do this, we introduce the concept of cooperative substitutes and complements as a convenient way of summarising how changes in one choice variable affect the marginal contribution of another to industry profits. This concept is potentially applicable to any context in which profits are maximised by choice of more than one variable. In this paper we use it to illuminate the conditions for a research joint venture to choose equal levels of R\&D by all member firms.

Section 2 defines cooperative substitutes and complements, derives the second-order conditions for symmetric cooperative outcomes in a general model, and relates them to those of d'Aspremont and Jacquemin and Salant and Shaffer. Section 3 applies our results to the linear-quadratic model of d'Aspremont and Jacquemin, and considers their implications for the desirability of allowing R\&D cooperation.

\footnotetext{
${ }^{1}$ Salant and Shaffer expand on their criticism of the literature on research joint ventures in their 1998 paper, and their analysis has already been quoted with approval in at least two textbooks. See Martin (2002, p. 453) and Shy (1996, p. 233).
} 


\section{Symmetric cooperative optima in a general $R \& D$ model}

Consider a two-stage game in which first-stage investment in R\&D by $n$ ex ante identical firms lowers their second-stage marginal costs of production. Let $\boldsymbol{x}=\left(x^{1}, \ldots\right.$, $x^{n}$ ) denote the vector of $\mathrm{R} \& \mathrm{D}$ investments. In this section we consider the cooperative case, where firms agree to coordinate their investment levels in order to maximise industry profits. ${ }^{2}$ We denote total industry profit by a scalar function $\Pi(\boldsymbol{x})=\sum_{k} \pi^{k}(\boldsymbol{x}){ }^{3}$ After the levels of R\&D are chosen cooperatively, firms compete in a standard noncooperative fashion, choosing either outputs or prices. The function $\Pi(\boldsymbol{x})$ takes into account the effects of R\&D levels on the profits that will be earned in the subsequent stage. $^{4}$

\subsection{Cooperative substitutes and complements}

It turns out that understanding the conditions for maximisation of industry profits is greatly helped by introducing a new concept which we call cooperative substitutability and complementarity. We define this as follows:

Definition. The levels of R\&D of firms $i$ and $j$ are cooperative substitutes if and only if the cross-derivative $\Pi_{i j}$ is negative, otherwise they are cooperative complements.

This definition is reminiscent of but not the same as the standard definition of strategic substitutes and complements due to Bulow et al. (1985). What is at issue here is the effect of one firm's R\&D on the marginal contribution of another firm's R\&D to industry profits. By contrast, the concept of strategic substitutes refers to the cross-effect of one firm's R\&D on the marginal profits of another firm..$^{5}$ Strategic substitutability and complementarity is the natural concept to use in a non-cooperative context, and we show below that the concept of cooperative substitutability and complementarity plays a similar role in the cooperative context.

Precise conditions for the criteria for cooperative and strategic substitutability to coincide are given in Appendix A. The most important case where this happens is that of a symmetric equilibrium with only two firms. Intuitively, this is because the criterion for strategic substitutability, $\pi_{i j}^{i}>0$, is inherently bilateral, even in an $n$-firm industry, since it involves the effect of one firm's R\&D on the marginal profitability of another's. By contrast, the criterion for cooperative substitutability, $\Pi_{i j}>0$, can be written as $\sum_{k} \pi_{i j}^{k}$,

\footnotetext{
${ }^{2}$ We follow much of the literature in using the terms "R\&D cooperation" and "research joint venture" interchangeably, to describe a situation where firms first choose their R\&D levels cooperatively and then compete in the second stage, enjoying the same cost spillovers per unit of rival R\&D as they would in the absence of cooperation. Kamien et al. (1992) refer to this as an "R\&D cartel", and use the term "RJV cartel" for the case where spillovers between cooperating firms are complete. The analysis of Section 2 applies to both cases.

${ }^{3}$ Superscripts indicate the firm in question, and subscripts will subsequently be used to denote partial derivatives. Thus $\Pi_{i}$ is the partial derivative of the industry profit function with respect to $x^{i}$, and $\Pi_{i j}$ is the partial derivative of $\Pi_{i}$ with respect to $x^{j}$.

${ }^{4}$ This general specification of second-stage competition follows Leahy and Neary (1997).

${ }^{5}$ Following Bulow et al. (1985, p. 494), $x^{i}$ is a strategic substitute for $x^{j}$ if and only if $\pi_{i j}^{i}$ is negative.
} 
which is inherently multilateral. Only in a symmetric 2-firm case do these criteria coincide. ${ }^{6}$

\subsection{Case I: ex ante equal treatment}

The simplest way to model a cooperative equilibrium between ex ante identical firms, and the one considered by d'Aspremont and Jacquemin (1988), is to confine attention to cases where all firms invest equally in $R \& D$. This is the natural starting point for examining cooperation on R\&D when side-payments between firms cannot be made. Since the firms remain independent entities, and engage in non-cooperative competition in the post-R\&D stage, it is stretching credulity to assume that any firm would enter a cooperative agreement which gave it lower profits than an ex ante identical rival firm. Hence, in the absence of side payments, we assume that all firms invest equally in R\&D or, following Salant and Shaffer (1999), that they are subject to "equal treatment".

Given the assumption of ex ante equal treatment, there is in effect only a single choice variable: the level of $R \& D$ common to all firms. In other words, we confine attention to $\mathrm{R} \& \mathrm{D}$ vectors in which all elements are equal, and seek that one which maximises industry profits. The effect of an arbitrary change in all the $x^{i}$ on industry profits is given by

$$
\mathrm{d} \Pi=\sum_{i}^{n} \Pi_{i} \mathrm{~d} x^{i}
$$

Since $x^{i}=x$ and $\mathrm{d} x^{i}=\mathrm{d} x$ for all $i$, the first-order condition for an interior optimum (i.e., one with $\left.x^{i}>0, \forall i\right)$ is given by:

$$
\frac{\mathrm{d} \Pi}{\mathrm{d} x}=\sum_{i}^{n} \Pi_{i}=n \Pi_{i}=0
$$

Clearly this is identical to setting $\Pi_{i}$ equal to zero. The second-order condition is then that no equiproportionate increase in the R\&D levels of all firms (including firm $i$ ) can raise $\Pi_{i}$. We state this formally as follows:

Proposition 1. When firms are treated equally ex ante, the sufficient second-order condition for a symmetric optimum is:

$$
\Pi_{i i}+(n-1) \Pi_{i j}<0, \quad \forall i, j, i \neq j
$$

This generalises the second-order condition first derived by d'Aspremont and Jacquemin (1988, p. 1134, footnote 7) in the two-firm linear-quadratic case. Provided Eqs. (2) and (3) hold, industry profits are maximised subject to the constraint that all firms have the same level of R\&D. Note that Eq. (3) gives the second-order condition in full. In particular, it is not necessary that the second derivative of industry profits with respect to the R\&D of a single firm, $\Pi_{i i}$, be negative.

\footnotetext{
${ }^{6}$ With only two firms, the criterion for cooperative substitutability becomes $\pi_{i j}^{i}+\pi_{i j}^{j}<0$. By Young's Theorem, $\pi_{i j}^{j}=\pi_{j i}^{j}$, and by symmetry $\pi_{j i}^{j}=\pi_{i j}^{i}$, so the criterion reduces to $2 \pi_{i j}^{i}<0$, which is equivalent to the criterion for strategic substitutability. See Appendix A for further details.
} 


\subsection{Case II: ex post equal treatment}

Although all firms have identical profit functions (reflecting the facts that they have identical technology and face identical demands), the research joint venture need not treat all members equally. As emphasised by Salant and Shaffer (1998), this could arise when side payments between firms are allowed, either via direct transfers or marketswapping agreements. ${ }^{7}$ However, the fact that unequal treatment is possible does not guarantee that it is optimal. In this section, we seek conditions under which an unconstrained maximisation will exhibit the same level of R\&D by all firms. We call this situation equal treatment ex post.

As before, the first-order condition for maximisation of industry profits $\Pi(x)$ by choice of $\boldsymbol{x}$ at a symmetric interior optimum is $\Pi_{i}=0$ for all $i$. The crucial difference from the last section comes in the second-order conditions, since now all elements of the R\&D vector can be changed independently. Let $\Pi_{x x}\left(x^{\circ}\right)$ be the square matrix of second-order partial derivatives evaluated at a symmetric interior optimum, $\boldsymbol{x}^{\mathrm{o}}$. The second-order sufficient condition is that $\Pi_{x x}\left(x^{o}\right)$ is the matrix of a negative definite quadratic form. Therefore, beginning with negative, the principal minors of the determinant $\left|\Pi_{x x}\left(\boldsymbol{x}^{\mathrm{o}}\right)\right|$ must alternate in sign. Let the $m$ th principal minor be obtained by deleting the last $n-m$ rows and columns of $\left|\Pi_{x x}\left(\boldsymbol{x}^{\mathrm{o}}\right)\right|$. Then the special structure of the matrix allows us to write the second-order condition in a compact form: ${ }^{8}$

Proposition 2. When firms are not treated equally ex ante, the sufficient second-order condition for a symmetric optimum is:

$$
(-1)^{m}\left(\Pi_{i i}-\Pi_{i j}\right)^{m-1}\left[\Pi_{i i}+(m-1) \Pi_{i j}\right]>0, \quad \forall m \leq n .
$$

This condition is not very transparent. However, its economic implications are much clearer when it is restated as follows:

Proposition 3. When firms are not treated equally ex ante, necessary and sufficient conditions for the second-order condition, Eq.(4), to hold at a symmetric optimum are given by:

$$
\begin{aligned}
& \Pi_{i i}+(n-1) \Pi_{i j}<0, \quad \forall i, j, i \neq j \\
& \Pi_{i i}-\Pi_{i j}<0 \quad \forall i, j, i \neq j
\end{aligned}
$$

Proof. To prove necessity, we need to show that Eq. (4) implies Eqs. (5) and (6). Consider therefore Eq. (4) for different values of $m$. Setting $m$ equal to one gives what we can call the no-own-deviation condition, $\Pi_{i i}<0$. Setting $m$ equal to two implies that $\Pi_{i i}^{2}>\Pi_{i j}^{2}$, which means that the absolute value of $\Pi_{i i}$ exceeds that of $\Pi_{i j}$. Combined with the noown-deviation condition, this implies condition (6). Finally, setting $m$ equal to $n(n \geq 2)$,

\footnotetext{
${ }^{7}$ The precise conditions under which such market-swapping agreements are sustainable have been investigated by Bernheim and Whinston (1990).

${ }^{8}$ See Dixit (1986), following Seade (1983).
} 
the first part of condition (4) becomes $(-1)^{n}\left(\Pi_{i i}-\Pi_{i j}\right)^{n-1}$, which from Eq. (6) is negative for all $n$. Hence, condition (4) as a whole implies condition (5).

Next, we need to prove sufficiency. Multiplying Eq. (6) by $n-1$ and adding to Eq. (5) gives the no-own-deviation condition, $\Pi_{i i}<0$. This plus Eq. (5) implies that $\Pi_{i i}+(m-1) \Pi_{i j}$ is negative for all $m \leq n$. Combining this with Eq. (6) implies condition (4).

Conditions (5) and (6) have a nice intuitive interpretation. For a symmetric choice of R\&D to maximise industry profits, it must not be possible for any one firm or any combination of firms to deviate profitably. Proposition 3 shows that Eqs. (5) and (6) are necessary and sufficient for the second-order conditions to hold, implying that any small deviation from a symmetric optimum can be expressed as a linear combination of only two primitive deviations.'

The first kind of primitive deviation is a uniform increase in the R\&D of all firms. This changes the first-order condition for optimal cooperative choice of R\&D by a typical firm, $\Pi_{i}=0$, by an amount equal to $\Pi_{i i}+(n-1) \Pi_{i j}$. To ensure that this deviation is unprofitable requires that condition (5) hold. We have already seen in Proposition 1 that this condition is sufficient for a symmetric optimum when firms are treated equally ex ante, the case considered by d'Aspremont and Jacquemin. A different interpretation can be given to this condition by recalling our definition of cooperative substitutability and complementarity. The condition in Eq. (5) implies that $\Pi_{i j}$ cannot exceed $-\Pi_{i i} /(n-1)$. Since $\Pi_{i i}$ must be negative at the optimum, this means that $\Pi_{i j}$ cannot be too positive, or, in words, that the optimum should not exhibit too much cooperative complementarity. Henceforward we call Eq. (5) the Restricted Cooperative Complementarity or RCC Condition. Too much cooperative complementarity would imply that a uniform increase in R\&D by all firms (a symmetric deviation from a symmetric equilibrium) would raise the marginal contribution to industry profits of every firm, implying that the initial allocation did not maximise industry profits.

The second kind of primitive deviation is a reallocation of R\&D between any group of firms, holding their total R\&D constant. Suppose, without loss of generality, that the group consists of the first $m$ firms, where $m$ can take any value between 2 and $n$. Using $\Delta x^{i}$ to denote the change in the $\mathrm{R} \& \mathrm{D}$ of firm $i$, we therefore have:

$$
\sum_{1}^{m} \Delta x^{j}=0 \text { and } \Delta x^{h}=0, m<h \leq n .
$$

We can see immediately that this change does not affect the marginal contribution to industry profits of the R\&D of a typical firm $h$ outside the group:

$$
\Delta \Pi_{h}=\sum_{j=1}^{m} \Pi_{h j} \Delta x^{j}=\Pi_{i j} \sum_{j=1}^{m} \Delta x^{j}=0, \quad m<h \leq n .
$$

Here we use the fact that, for small deviations from a symmetric equilibrium, all the crossderivatives are equal: $\Pi_{h j}=\Pi_{i j}$, for all $h, i$ and $j$. Next, consider the effect on the marginal

\footnotetext{
${ }^{9}$ Note that the no-own-deviation condition, $\Pi_{i i}<0$, is not part of the conditions in Proposition 3, since it is implied both by Eq. (4) and by Eqs. (5) and (6).
} 
contribution to industry profits of a firm in the group which increases its R\&D. (Similar arguments apply, mutatis mutandis, to a firm which reduces its R\&D.) The first-order condition for the $\mathrm{R} \& \mathrm{D}$ of firm $i, \Pi_{i}=0$, changes by an amount equal to:

$$
\begin{aligned}
\Delta \Pi_{i} & =\Pi_{i i} \Delta x^{i}+\sum_{j \neq i}^{m} \Pi_{i j} \Delta x^{j} \\
& =\Pi_{i i} \Delta x^{i}+\Pi_{i j} \sum_{j \neq I}^{m} \Delta x^{j} \\
& =\left(\Pi_{i i}-\Pi_{i j}\right) \Delta x^{i}
\end{aligned}
$$

where the final step uses Eq. (7). To ensure that this deviation reduces firm $i$ 's marginal contribution to industry profits requires that the term in brackets be negative, i.e., that condition (6) hold. This implies that $\Pi_{i j}$ cannot be less than $\Pi_{i i}$. This means that $\Pi_{i j}$ cannot be too negative, or, in words, that the optimum should not exhibit too much cooperative substitutability. Hence we refer to this as the Restricted Cooperative Substitutability or RCS Condition. Too much cooperative substitutability would imply that, following a reallocation of $R \& D$ between a group of firms (an asymmetric deviation from a symmetric equilibrium), the fall in R\&D by some of those firms would raise the marginal contribution to industry profits of firms that increased their R\&D sufficiently to offset the negative own effect on marginal profitability $\Pi_{i i}$, implying that the initial allocation did not maximise industry profits.

\subsection{The Salant-Shaffer approach: no two-firm deviations allowed}

We next want to explore how the second-order conditions derived in the last section relate to the approach of Salant and Shaffer (1999). They do not explicitly discuss secondorder conditions for a symmetric optimum. Instead, they explore the conditions under which a small deviation from a symmetric optimum by two firms, keeping their total R\&D fixed, leads to an increase in industry profits. In this section, we focus on Salant and Shaffer's central analytic contribution, a sufficient condition for optimal first-stage behaviour to be asymmetric when asymmetries in marginal costs are costly to introduce, given by Eq. (7) in their paper. We first derive the Salant-Shaffer condition and then relate it to the second-order conditions for a symmetric optimum derived in the last section. ${ }^{10}$

Following Salant and Shaffer, we seek a condition for a reallocation of investment between two firms, keeping the sum of marginal costs constant, to raise industry profits. Consider what they call the "iso-sum locus", $x^{2}=g\left(x^{1} ; x^{*}\right)$, which gives combinations of $x^{1}$

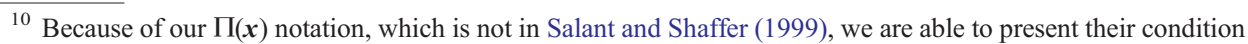
in a more compact and general form than in their paper. Whereas they restrict attention to homogeneous-product Cournot competition, our results also apply, as already noted, when goods are symmetrically differentiated and when firms compete in either a Cournot or Bertrand manner.
} 
and $x^{2}$ along which the sum of marginal costs is constant, when all $n-2$ other firms keep their R\&D levels at a symmetric level $x^{*}$. When firm 1 invests $x^{1}$, firm 2 invests $x^{2}=g\left(x^{1}\right.$; $\left.x^{*}\right)$ and the other $n-2$ firms invest $x^{*}$, industry profits can be written as:

$$
\tilde{\Pi}\left(x^{1} ; x^{*}\right)=\Pi\left[x^{1}, g\left(x^{1} ; x^{*}\right), x^{*}, \ldots, x^{*}\right] .
$$

Differentiating Eq. (10) with respect to $x^{1}$ and evaluating at the symmetric R\&D vector $\boldsymbol{x}^{*}$ gives:

$$
\tilde{\Pi}^{\prime}\left(x^{*} ; x^{*}\right)=\Pi_{1}\left(\boldsymbol{x}^{*}\right)+g^{\prime} \Pi_{2}\left(\boldsymbol{x}^{*}\right)=0,
$$

The expression in Eq. (11) is zero since $\Pi_{1}\left(x^{*}\right)=\Pi_{2}\left(x^{*}\right)$ (because $x^{*}$ is symmetric) and $g^{\prime}\left(x^{*} ; x^{*}\right)=-1$ (as shown by Salant and Shaffer). This is a local minimum when:

$$
\tilde{\Pi}^{\prime \prime}\left(x^{*} ; x^{*}\right)=\Pi_{11}\left(x^{*}\right)+g^{\prime}\left[\Pi_{12}\left(x^{*}\right)+\Pi_{21}\left(x^{*}\right)\right]+\left(g^{\prime}\right)^{2} \Pi_{22}\left(x^{*}\right)+g^{\prime \prime} \Pi_{2}\left(x^{*}\right)>0 .
$$

This expression can be simplified since $\Pi_{11}\left(x^{*}\right)=\Pi_{22}\left(x^{*}\right)$ (because $x^{*}$ is symmetric) and $\Pi_{12}\left(\boldsymbol{x}^{*}\right)=\Pi_{21}\left(\boldsymbol{x}^{*}\right)$. As in Salant and Shaffer, denote by $\boldsymbol{x}^{\Pi}=\left(x^{\Pi}, \ldots, x^{\Pi}\right)$ the investment combination that maximises industry profit subject to the ex ante equaltreatment constraint that all firms must invest equally. Then the Salant and Shaffer condition can be written as:

$$
\tilde{\Pi}^{\prime \prime}\left(x^{\Pi} ; x^{\Pi}\right)=2\left[\Pi_{11}\left(\boldsymbol{x}^{\Pi}\right)-\Pi_{12}\left(\boldsymbol{x}^{\Pi}\right)\right]+g^{\prime \prime} \Pi_{2}\left(\boldsymbol{x}^{\Pi}\right)>0 .
$$

When this condition holds, a deviation from symmetry raises industry profits.

We now show that the Salant-Shaffer condition is incompatible with the second-order condition:

Proposition 4. The Salant-Shaffer condition, Eq.(13), is the converse of the RCS condition, Eq.(6), at a symmetric interior equilibrium.

Proof. The proof is immediate from inspection of Eq. (13). Since the choice of firms 1 and 2 was arbitrary, we can reexpress Eq. (13) in terms of any two firms $i$ and $j$. At the optimum, $\Pi_{2}$ is zero, and so Eq. (13) can hold if and only if Eq. (6) does not hold.

It is straightforward to extend this analysis to the maximisation of social surplus which Salant and Shaffer also consider in section II.B of their paper. Here too the Salant-Shaffer condition and the second-order condition for a welfare maximum cannot both hold at a symmetric interior equilibrium.

While we have shown that Eqs. (13) and (4) are incompatible, it does not follow that a violation of Eq. (13) implies that Eq. (4) holds. A violation of the Salant-Shaffer condition is necessary but not sufficient for the second-order condition to hold, as our Proposition 3 shows. The Salant and Shaffer approach is to consider movements away from a symmetric equilibrium on a path along which the investments of all but two firms are constant. But there is no justification for restricting attention to such paths in an $n$-firm problem. What is required instead is to consider all possible deviations from a candidate optimum, and this is exactly what the standard second-order condition does. 


\section{The d'Aspremont and Jacquemin case}

In this section we show how the general results of the previous section specialise to the linear-quadratic example first explored by d'Aspremont and Jacquemin. Focusing on an explicit example allows us to relate the general results to underlying parameters in a more transparent way. It also allows us to address the quantitative importance of the different conditions. Our strategy is to derive the second-order conditions for a symmetric cooperative equilibrium and then to show how they relate to the Salant-Shaffer condition and to the stability condition for the non-cooperative equilibrium.

\subsection{The model}

As in d'Aspremont and Jacquemin, Salant and Shaffer (1998) and many other papers, we consider a homogeneous-product duopoly where firms first engage in cost-reducing R\&D and then in Cournot competition. With $n$ firms, the model is most easily solved by working with variables for a typical firm and for the industry as a whole: we denote these by $x^{i}$ and $X \equiv \sum_{k} x^{k}$ respectively for R\&D, and by $q^{i}$ and $Q \equiv \sum_{k} q^{k}$ for output. Otherwise the model is standard. Costs of $\mathrm{R} \& \mathrm{D}$ are quadratic, equal to $\gamma\left(x^{i}\right)^{2} / 2$ for firm $i$. Production costs are independent of output and fall linearly in own and rival R\&D. This in turn implies that they are decreasing in a weighted average of own and industry R\&D:

$$
\begin{aligned}
c^{i} & =c_{0}-\theta\left(x^{i}+\beta \sum_{k \neq i} x^{k}\right) \\
& =c_{0}-\theta\left[(1-\beta) x^{i}+\beta X\right]
\end{aligned}
$$

where $\beta$ is the spillover coefficient. Finally, demand is linear: $p=a-b Q$. We assume that $A \equiv a-c_{0}>0,0 \leq \beta \leq 1$ and $\gamma>0$.

In the second stage, firms choose outputs independently to maximise operating profits $\left(p-c^{i}\right) q^{i}$. This yields first-order conditions which equate marginal revenue to marginal cost for each firm: $p-b q^{i}=c^{i}$. Summing over all firms we can solve for industry output as a function of industry R\&D:

$$
Q=\frac{1}{(n+1) b}[n A+\theta\{1+(n-1) \beta\} X]
$$

This in turn allows us to solve for the output of firm $i$, which is always increasing in own R\&D but increasing in rival R\&D (i.e., industry $R \& D$ for given $x^{i}$ ) only for high spillovers $(\beta>1 / 2)$ :

$$
q^{i}=\frac{1}{(n+1) b}\left[A+\theta(n+1)(1-\beta) x^{i}+\theta(2 \beta-1) X\right]
$$

In the first stage, the RJV chooses the levels of R\&D to maximise industry profits:

$$
\Pi=\sum_{k=1}^{n} \pi^{k}, \text { where } \pi^{k}=b\left(q^{k}\right)^{2}-\frac{1}{2} \gamma\left(x^{k}\right)^{2}
$$


This yields the first-order conditions for R\&D:

$$
\Pi_{i}=\frac{2}{n+1} \theta\left[(n+1)(1-\beta) q^{i}+(2 \beta-1) Q\right]-\gamma x^{i}=0, \quad i=1, n
$$

These can then be solved for the equilibrium values of R\&D.

\subsection{Second-order conditions at a symmetric equilibrium}

If the RJV is to maximise profits, the second-order conditions with respect to R\&D levels must hold at the optimum. Let us assume now that the equilibrium is symmetric. To find the second-order conditions, specialise the general expressions from the last section to the linear-quadratic case and then evaluate them using the derivatives of Eq. (18). The detailed derivations are given in Appendix B. We show there that $\Pi_{i j}$ is negative, and so R\&D levels are cooperative substitutes, if and only if the spillover parameter is less than a half. As for the RCC condition (Eq. (5) specialised to the linear-quadratic case), it becomes: ${ }^{11}$

$$
\Pi_{i i}+(n-1) \Pi_{i j}<0 \Leftrightarrow \eta<\frac{1}{2}\left[\frac{n+1}{1+(n-1) \beta}\right]^{2}
$$

Here, following Leahy and Neary (1996), we use $\eta$ to denote $\theta^{2} / b \gamma$, which can be interpreted as the relative effectiveness of $R \& D$. Finally, the RCS condition, Eq. (6), becomes:

$$
\Pi_{i i}-\Pi_{i j}<0 \Leftrightarrow \eta<\frac{1}{2(1-\beta)^{2}}
$$

Note that this is independent of $n$.

Figs. 1 and 2 illustrate conditions (19) and (20) in $\{\eta, \beta\}$ space, for the case of two firms $(n=2) .{ }^{12}$ Both conditions can be explained intuitively in terms of the trade-off between the relative effectiveness of $R \& D$ and the degree of cooperative substitutability. An increase in $\eta$ makes it more likely that both parts of the second-order conditions will be violated. An increase in $\beta$ raises the degree to which $\mathrm{R} \& \mathrm{D}$ levels are cooperative complements. Hence, starting from a point where the RCC condition just holds, an increase in $\eta$ must be compensated by a fall in $\beta$ (i.e., a decrease in cooperative complementarity) to restore the condition. Thus the locus corresponding to the RCC condition is downward sloping in Fig. 1. By contrast, starting from a point where the RCS condition just holds, an increase in $\eta$ must be offset by an increase in $\beta$, and so the locus is upward-sloping.

Given the configuration of the loci in Fig. 1, we can now consider the different regions in Fig. 2. In Region A, above the locus corresponding to the RCC condition, an equiproportionate increase in $\mathrm{R} \& \mathrm{D}$ levels raises profits without bound. Hence this region

\footnotetext{
${ }^{11}$ Because of the special functional forms used, both parts of the second-order conditions depend on parameters only. Hence if they hold at one point they must hold at all. By contrast, in the general model of Section 2, the local results need not hold globally.

12 Salant and Shaffer (1998) present similar diagrams drawn in $\{1 / \eta, \beta\}$ space. Our perspective seems more natural since the vertical axis is bounded at zero, the case where R\&D is either prohibitively expensive or has no effect on production costs, and so its equilibrium level is always zero.
} 


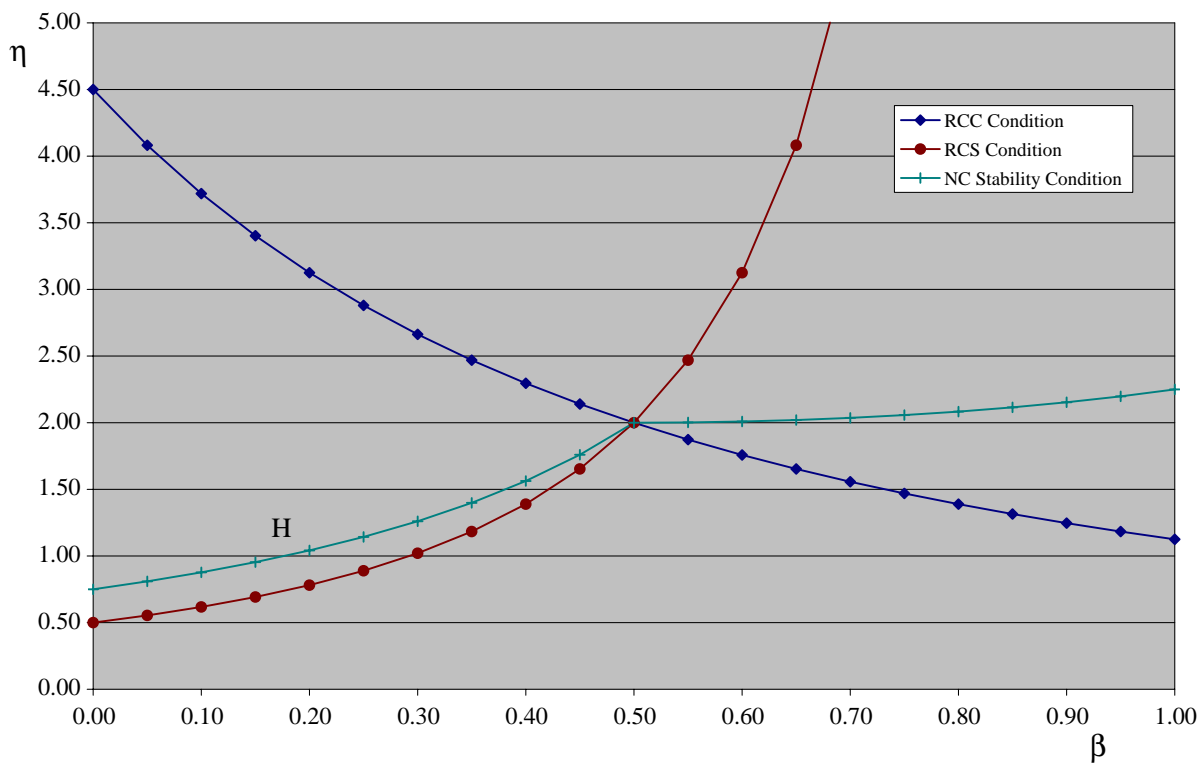

Fig. 1. Second-order conditions for a symmetric RJV.

of parameter space is not economically meaningful. By contrast, regions $\mathrm{B}$ and $\mathrm{C}$ (the difference between them to be considered in the next section) are consistent with a symmetric optimum if the firms are treated equally ex ante. However, if they are not, then

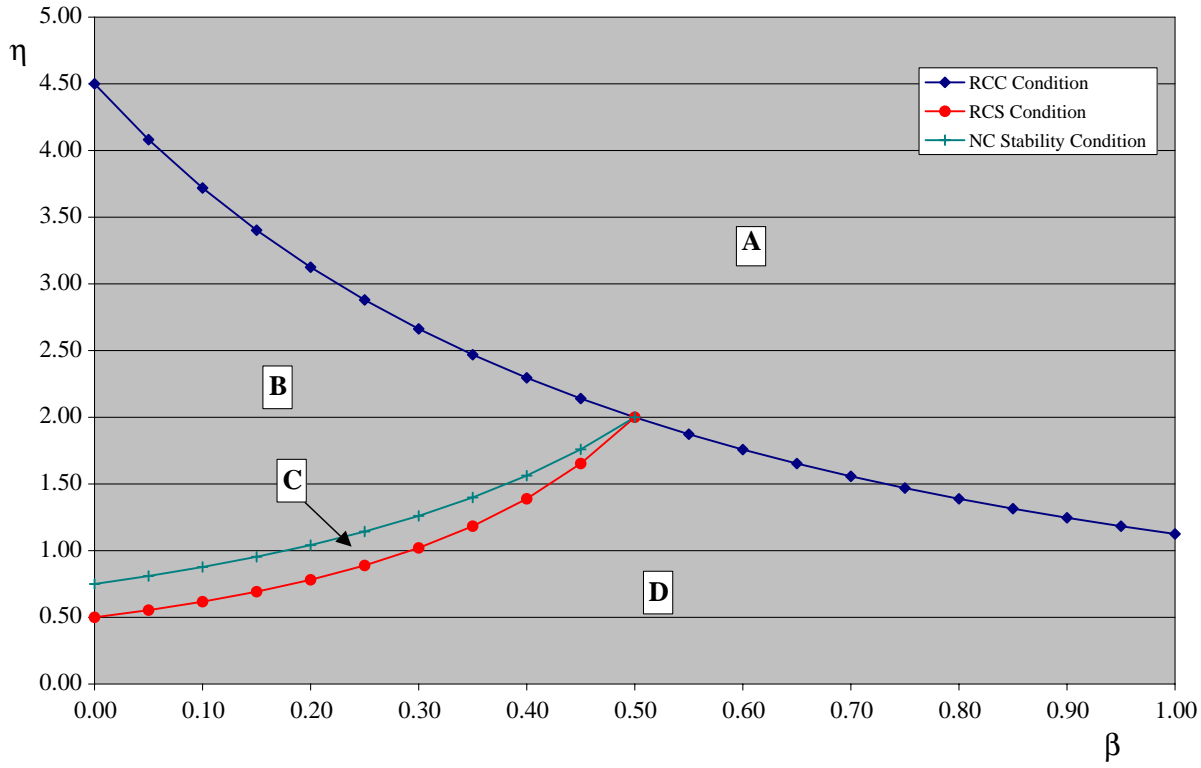

Fig. 2. Admissible regions for a symmetric RJV. 
the symmetric equilibrium does not satisfy the second-order conditions, since profits can be increased by reallocating $R \& D$ between the two firms. In that case, as Salant and Shaffer have argued, the pattern of R\&D levels which maximises profits for the RJV is asymmetric. Finally, in Region D the optimum is symmetric irrespective of whether or not firms are treated equally ex ante.

\subsection{Comparison with the non-cooperative equilibria}

The preceding discussion might suggest that asymmetric RJV's are important for a wide range of parameters. However, we have to recall that this issue is of theoretical and policy interest in the first place only because of the potential role of RJV's in leading to improved outcomes relative to non-cooperative equilibria. For the comparison to be meaningful, the non-cooperative equilibrium itself must be a meaningful one. In particular, it should satisfy the standard stability conditions for a non-cooperative Nash equilibrium. ${ }^{13}$

The stability conditions for this game have been derived in Leahy and Neary (1997, Appendix), drawing on Dixit (1986), Hahn (1962) and Seade (1980). As shown there, for $\beta$ less than a half, stability of the non-cooperative game requires that the following condition hold:

$$
\beta<1 / 2: \quad \pi_{i i}^{i}-\pi_{i j}^{i}<0 \Leftrightarrow \eta<\frac{n+1}{2(1-\beta)[n-(n-1) \beta]}
$$

Following our earlier discussion of the cooperative case, we can call this condition "Restricted Strategic Substitutability". Conversely, for $\beta$ greater than a half, stability requires a different condition which we can call "Restricted Strategic Complementarity": ${ }^{14}$

$$
\beta>1 / 2: \pi_{i i}^{i}+(n-1) \pi_{i j}^{i}<0 \Leftrightarrow \eta<\frac{(n+1)^{2}}{2[1+(n-1) \beta][n-(n-1) \beta]}
$$

These conditions are illustrated for $n$ equal to two in Figs. 1 and $2 .^{15}$ The small region denoted by $\mathrm{C}$ in Fig. 2 shows the parameter combinations which violate the RCS condition but satisfy the non-cooperative stability condition. Only in region $\mathrm{C}$ can asymmetric cooperative equilibria be compared with symmetric non-cooperation.

For higher values of $n$, the conclusions from Fig. 2 are reinforced. As already noted, the RCS condition, the boundary between regions $\mathrm{C}$ and $\mathrm{D}$, is independent of $n$. By contrast, as $n$ rises the RCC condition becomes more demanding for $\beta$ greater than a half. From Eq. (19), an increase in $n$ reduces the permissible level of cooperative complementarity, leading the curve to pivot clockwise around the point where $\beta$ equals a half. As a result region $A$ expands at the expense of region D. Finally, the curve representing the non-

\footnotetext{
${ }^{13}$ An alternative interpretation, explored by Amir and Wooders (1998), is to assume that, when the symmetric Nash equilibrium in R\&D is unstable, the outcome will be an asymmetric equilibrium.

14 These conditions are both necessary and sufficient when $n$ is even. When $n$ is odd, condition (21) is only sufficient for stability.

${ }^{15}$ Henriques (1990) was the first to explore the stability of non-cooperative equilibria in this model. The case corresponding to the parameter values considered by her is denoted by point $\mathrm{H}$ in Fig. 1.
} 
cooperative stability condition (21) falls as $n$ rises, squeezing region C. In the limit, as $n$ becomes very large, Eq. (21) coincides with Eq. (20) so this region vanishes, and there are no parameter combinations where a symmetric non-cooperative equilibrium can be compared with an asymmetric RJV.

\section{Conclusion}

In this paper we have considered the choice of R\&D levels in an industry where firms cooperate to form a research joint venture. We introduce the concept of cooperative substitutes and complements, and use it to throw light on the conditions for a research joint venture to choose equal levels of R\&D for all member firms. Our principal result, Proposition 3, shows that the second-order condition for a symmetric choice of $R \& D$ is equivalent to requiring that only two kinds of deviation from the optimum are unprofitable. First is a uniform increase in the R\&D of all firms. This is the sole deviation which must be unprofitable if firms are required to be treated equally ex ante, perhaps because side payments are ruled out, the case considered by d'Aspremont and Jacquemin (1988). For this deviation to be unprofitable requires a restriction on the degree of cooperative complementarity. The second kind of deviation which must be unprofitable is a reallocation of R\&D between an arbitrary subset of firms, holding constant their total level of R\&D. For this deviation to be unprofitable requires a restriction on the degree of cooperative substitutability, which we show is the converse of the condition derived by Salant and Shaffer (1999) in the case of a twofirm deviation.

In addition to considering the conditions for a profit-maximising cooperative outcome to be symmetric, we compare these conditions with those (already in the literature) for a non-cooperative equilibrium to be stable. In so far as these models are relevant to policy, it is because they throw light on the issue of whether cooperative research joint ventures should be allowed in preference to a non-cooperative oligopolistic equilibrium. Hence it is also necessary to consider the circumstances in which such an equilibrium makes sense, by checking its second-order and stability conditions (see Henriques, 1990 and Amir and Wooders, 1998). We show that, for a widely used example, the comparison between an asymmetric cooperative outcome and a symmetric non-cooperative equilibrium is relevant for a very small range of parameters.

How do our results relate to the critique of the literature on $R \& D$ cooperation presented by Salant and Shaffer $(1998,1999)$ ? Whereas they claim to find "error" in much of the literature on two-stage investment games (including our 1997 paper), we do not assert that their specific statements are false. ${ }^{16}$ Rather, we show that they are true but incomplete. In

\footnotetext{
${ }^{16}$ Our criticisms apply to Salant and Shaffer's Sections II.B and III and not to their Section II.A, which discusses games where asymmetries in marginal costs are costless (such as learning by doing or resource extraction with depletion effects). Clearly a symmetric equilibrium is not profit-maximising in such models (since it violates the second-order condition), and Salant and Shaffer do not present any examples from the literature which suggest otherwise.
} 
particular, whereas they derive a sufficient condition for a two-firm deviation from the optimum to be profitable, we show that all possible deviations from a candidate symmetric optimum can be expressed as combinations of only two primitive deviations, one of which is the Salant-Shaffer type.

Where we disagree with Salant and Shaffer is in the interpretation of their results, as for example when they say of research joint ventures: "there is a strong presumption that nonidentical actions by identical agents in the first stage are required to maximize social surplus and industry profit." (Salant and Shaffer, 1999, p. 586, emphasis in original). But in models where asymmetries in marginal costs are costly, there is no presumption that symmetric outcomes will fail to maximise profits or social welfare. On the contrary, they will do so locally, provided the objective function is sufficiently concave in the neighbourhood of the symmetric optimum, which in these models usually means, provided the investment or $\mathrm{R} \& \mathrm{D}$ cost function is sufficiently convex. How much convexity is required depends on the second-order conditions, no more and no less. Of course, to determine whether any point is a global optimum requires information about the objective function everywhere, but the Salant-Shaffer condition which (like the second-order conditions as a whole) is evaluated locally has nothing new to say on this. Hence, provided the appropriate second-order conditions are satisfied, all the conclusions in the extensive literature on research joint ventures are immune to the criticisms of Salant and Shaffer.

In conclusion, the concept of cooperative substitutes which we have introduced is likely to have other applications. Here we have considered only the case where cooperation occurs in the first stage of a two-stage game. However, it is also relevant in cases where cooperation is permanent, such as multi-plant or multi-product firms. For example, economies of scope in production of two goods by a multi-product firm tends to make them cooperative complements, although this effect may be offset if they are also substitutes in demand. In addition, while we have considered only the case where the cooperative includes all firms in the industry, it is also relevant to cooperation between a subset of firms. In that case the concept of cooperative substitutes is relevant to allocation within the cooperative, whereas the standard concept of strategic substitutes is relevant to external competition.

\section{Acknowledgements}

This research forms part of the International Trade and Investment Programme of the Geary Institute at UCD. We are grateful to Doug Bernheim, Steve Salant, Greg Shaffer and an anonymous referee for helpful comments.

\section{Appendix A. Cooperative versus strategic substitutes and complements}

Unlike the definition of strategic substitutes and complements, the definition of cooperative substitutes and complements is symmetric: if $x_{i}$ is a cooperative substitute 
for $x_{j}$, then the converse is also true. To determine the relationship between the two types of substitutability and complementarity, recall that industry profits equal the sum of the profits of all $n$ firms: $\Pi=\sum_{k} \pi^{k}$. Differentiating with respect to the R\&D of firm $i$ gives:

$$
\Pi_{i}=\sum_{k} \pi_{i}^{k}
$$

In a symmetric equilibrium, this simplifies to:

$$
\Pi_{i}=\pi_{i}^{i}+(n-1) \pi_{j}^{i}
$$

(where we use the fact that $\pi_{i}^{j}=\pi_{j}^{i}$ ). The own second derivative of industry profits therefore becomes:

$$
\Pi_{i i}=\sum_{k} \pi_{i i}^{k}
$$

or with symmetry:

$$
\Pi_{i i}=\pi_{i i}^{i}+(n-1) \pi_{j j}^{i}
$$

Finally, the cross derivative of industry profits, which is negative if and only if $R \& D$ levels are cooperative substitutes, equals:

$$
\Pi_{i j}=\sum_{k} \pi_{i j}^{k}
$$

or with symmetry:

$$
\Pi_{i j}=2 \pi_{i j}^{i}+(n-2) \pi_{i j}^{h}
$$

To derive Eq. (28) from Eq. (27), we use the facts that $\pi_{i j}^{i}=\pi_{j i}^{j}$ from symmetry; that $\pi_{i j}^{k}=\pi_{i j}^{h}$ for all $h$ and $k$ not equal to $i$ or $j$, from symmetry; and that $\pi_{j i}^{j}=\pi_{i j}^{j}$ from Young's Theorem. Crucially, however, $\pi_{i j}^{i}$ is not equal to $\pi_{i j}^{h}$ when $h, i$ and $j$ are all different. This is true even in the linear-quadratic case: see below.

Eq. (28) shows that the criteria for the two forms of substitutability do not coincide, even when we consider only symmetric equilibria. They coincide in two special cases. The first is where there are only two firms. In that case the final term in Eq. (28) vanishes and the criterion for cooperative substitutes, $\Pi_{i j}<0$, has the same sign as that for strategic substitutes, $\pi_{i j}^{i}<0$, irrespective of the functional forms and of the nature of competition. The second is the linear-quadratic model of Section 3, where, for all $n$, R\&D levels are both cooperative and strategic substitutes if and only if $\beta$ is less than one half. To see this, consider first the criterion for strategic substitutability, obtained by differentiating twice the expression in Eq. (17) for a single firm's profits. This gives:

$$
\pi_{i j}^{i}=\frac{2}{(n+1)^{2}} \eta \gamma(2 \beta-1)[n-(n-1) \beta]
$$


This is clearly negative if and only if $\beta$ is less than one half. Next, a similar direct calculation leads to the effects of a change in one firm's R\&D on the responsiveness of a second firm's profits to a change in a third firm's R\&D:

$$
\pi_{i j}^{h}=\frac{2}{(n+1)^{2}} \eta \gamma(2 \beta-1)^{2}
$$

Surprisingly, this is always positive, strictly so except when $\beta$ equals a half. (Note also that it differs from Eq. (29).) Finally, substituting from Eqs. (29) and (30) into Eq. (28) gives the expression for $\Pi_{i j}$ derived by a different route in Eq. (32) below. Like Eq. (29), this is negative if and only if $\beta$ is less than one half, confirming that the criteria for strategic and cooperative substitutability coincide in this case.

\section{Appendix B. Second-order conditions in the linear-quadratic case}

It turns out to be most convenient to derive the cross second derivative first. Inspecting the first-order condition (18) and the expressions for industry and firm output (Eqs. (15) and (16)), we can see that the cross derivative is simply the partial derivative of $\Pi_{i}$ with respect to industry $\mathrm{R} \& \mathrm{D} X$. Thus differentiating Eq. (18) gives:

$$
\Pi_{i j}=\frac{\partial \Pi_{i}}{\partial X}=\frac{2}{n+1} \theta\left[(n+1)(1-\beta) \frac{\partial q^{i}}{\partial X}+(2 \beta-1) \frac{\mathrm{d} Q}{\mathrm{~d} X}\right]
$$

Calculating $\partial q^{i} / \partial X$ and $\mathrm{d} Q / \mathrm{d} X$ from Eqs. (16) and (15), respectively, and substituting into Eq. (31) gives the cooperative substitutability term:

$$
\Pi_{i j}=\frac{2}{(n+1)^{2}} \eta \gamma(2 \beta-1)[n+2(1-\beta)]
$$

This is clearly negative, and so R\&D levels are cooperative substitutes, if and only if $\beta$ is less than a half, as stated in the text.

Next the own second derivative, which must be negative for the no-own-deviation condition to hold, can be written as follows:

$$
\Pi_{i i}=\frac{\partial \Pi_{i}}{\partial x^{i}}+\frac{\partial \Pi_{i}}{\partial X}=2 \theta(1-\beta) \frac{\partial q^{i}}{\partial X}-\gamma+\Pi_{i j}
$$

Subtracting Eq. (31) from this and substituting for $\partial q^{i} / \partial X$ gives the RCS condition:

$$
\Pi_{i i}-\Pi_{i j}=2 \eta \gamma(1-\beta)^{2}-\gamma<0
$$

Finally the RCC condition can be calculated in a similar way:

$$
\Pi_{i i}+(n-1) \Pi_{i j}=2 \eta \gamma\left[\frac{1+(n-1) \beta}{n+1}\right]^{2}-\gamma<0
$$




\section{References}

Amir, Rabah, Wooders, John, 1998. Cooperation vs. competition in R\&D: the role of stability of equilibrium. Journal of Economics 67, 63-73.

Bergstrom, Theodore C., Varian, Hal R., 1985. When are Nash equilibria independent of the distribution of agents' characteristics? Review of Economic Studies 52, 715-718.

Bernheim, Douglas B., Whinston, Michael D., 1990. Multimarket contact and collusive behavior. Rand Journal of Economics 21, 1-26.

Bulow, Jeremy I., Geanakoplos, John D., Klemperer, Paul D., 1985. Multimarket oligopoly: strategic substitutes and complements. Journal of Political Economy 93, 488-511.

d'Aspremont, Claude, Jacquemin, Alexis, 1988 (December). Cooperative and noncooperative R\&D in duopoly with spillovers. American Economic Review 78, 1133-1137.

Dixit, Avinash, 1986 (February). Comparative statics for oligopoly. International Economic Review 27, $107-122$.

Hahn, Frank H., 1962. The stability of the Cournot oligopoly solution. Review of Economic Studies 34, 329-331.

Henriques, Irene, 1990. Cooperative and noncooperative R\&D in duopoly with spillovers: comment. American Economic Review 80, 638-640.

Kamien, Morton I., Muller, Eitan, Zang, Israel, 1992. Research joint ventures and R\&D cartels. American Economic Review 82, $1293-1306$.

Leahy, Dermot, Neary, J. Peter, 1996. International R\&D rivalry and industrial strategy without government commitment. Review of International Economics 4, 322-338.

Leahy, Dermot, Neary, J. Peter, 1997. Public policy towards R\&D in oligopolistic industries. American Economic Review 87, 642-662.

Martin, Stephen, 2002. Advanced Industrial Economics, Second edition. Basil Blackwell, Oxford.

Salant, Stephen W., Shaffer, Greg, 1998. Optimal asymmetric strategies in research joint ventures. International Journal of Industrial Organization 16, 195-208.

Salant, Stephen W., Shaffer, Greg, 1999. Unequal treatment of identical agents in Cournot equilibrium. American Economic Review 89, 585-604.

Seade, Jesús, 1980. The stability of Cournot revisited. Journal of Economic Theory 23, 15-27.

Seade, Jesús, 1983. Prices, profits and taxes in oligopoly, Working paper, University of Warwick, 1983.

Shy, Oz, 1996. Industrial Organization: Theory and Applications. MIT Press, Cambridge, MA.

Van Long, Ngo, Soubeyran, Antoine, 1999. Asymmetric contribution to research joint ventures. Japanese Economic Review 50, 122-137. 